\title{
Fourier-Transform Infrared Absorption Spectroscopy in Binary Hydrocarbon-Alcohol Single Droplet Evaporation
}

\author{
Shane R. Daly, Nicholas Olson, and Christopher L. Hagen \\ Energy Systems Laboratory, School of Mechanical, Industrial and Manufacturing Engineering, Oregon State University, \\ 2600 NW College Way, Bend, OR 97703, USA
}

Correspondence should be addressed to Christopher L. Hagen; chris.hagen@oregonstate.edu

Received 20 August 2015; Revised 17 December 2015; Accepted 14 January 2016

Academic Editor: Stephane Schilt

Copyright (C) 2016 Shane R. Daly et al. This is an open access article distributed under the Creative Commons Attribution License, which permits unrestricted use, distribution, and reproduction in any medium, provided the original work is properly cited.

Broadband absorption spectroscopy, by way of FTIR, was used to investigate the vapor cloud of a single millimeter sized liquid droplet suspended by a syringe as it evaporates at standard conditions. Single beam data were collected every 8 seconds resulting in a time-resolved record. Species concentrations were tracked using their resonant absorption peaks and correlated with a multidimensional numerical model. The numerical model combined a Gaussian beam transmission through a temporally changing spherical vapor cloud with radial concentration gradients, informed by the $D^{2}$ law and interpreted using the Abel transform. There was fair agreement with temporal evaporation trends for single component runs. Multicomponent experiments of ethanol and isooctane showed synergistic blending effects and preferential evaporation of ethanol. Droplets were also suspended by a thermocouple to track the droplet temperature over time as they were subject to evaporative cooling. This work is the foundation of a basic technique for collecting useful data to inform a complex transport problem.

\section{Introduction}

Droplet research is a widely diverse topic with one application being the investigation of fuel evaporation in direct injected internal combustion engines. Combustion scientists are interested in the burning and transport behavior of hydrocarbon droplets because of the implications on emissions and fuel efficiency.

A significant body of fuel spray droplet research, both experimental and modeling, exists for combustion scientists $[1,2]$. However, single droplet fuel evaporation of mixtures is also of interest to help inform the spray work by extrapolating the behavior of one droplet to the aggregate behavior of many. For example, Wei et al. suspended droplet mixtures of propanol, methanol, and acetone using quartz optical fibers and conducted ultraviolet- (UV-) visible absorption spectroscopy in tandem with charge-coupled device (CCD) camera video [3]. Ghassemi et al. conducted a study of single droplet binary mixtures of heptane and hexadecane at elevated temperatures and pressures [4]; in their work, the droplets were suspended using a quartz fiber and their evaporation was recorded with a high-speed CCD camera. Hallett and Beauchamp-Kiss also used quartz fiber suspension and a camera to study single droplets of multicomponent fuels for correlating fuel distillation curves to likely composition [5]. Others, such as Arnold et al. and Grader et al., have electrodynamically trapped (suspended) single aerosol particles in tightly controlled environments and modulated the size of the particles by heating the micron sized targets with focused thermal IR sources [6, 7]. In these works, particle size was monitored by secondary laser sources and scattering techniques.

The modeling complement to single droplet evaporation experiments can be minimally accomplished by way of the $D^{2}$ law method [8]. That said, much more complex models exist. See Raghuram and Raghavan, Camm et al., or Hołyst et al. for more involved single droplet numerical representations [9-11]. We present here a multidimensional model which is more elaborate than, albeit rooted in, the $D^{2}$ law.

\section{Theory and Approach}

The work done here is a step towards identifying preferential vaporization (different components evaporating at different 


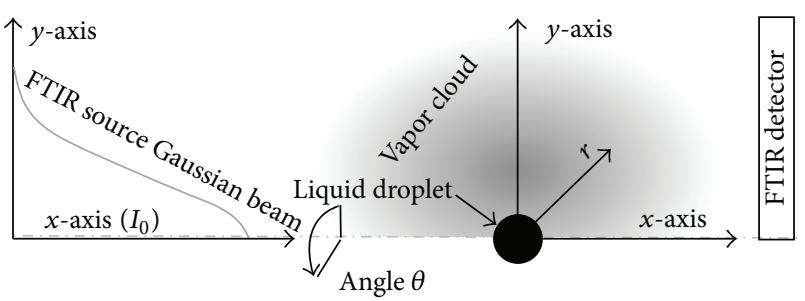

FIGURE 1: Two-dimensional representation of the absorption process used to derive the model presented here. Note that $\theta$ is the angle of revolution and that the beam moves from left to right in this depiction.

times) of a binary oxygenated hydrocarbon droplet in an effort to improve understanding of mixture behavior. Our work highlights the study of a single relatively large droplet $(1.9[\mathrm{~mm}]$ diameter) under ambient conditions. This droplet size has been investigated in similar experiments, as mentioned, by Ghassemi et al. (1.1-1.3 [mm] diameter) and Hallett and Beauchamp-Kiss (1.4-1.8 [mm] diameter). Our droplets were probed by infrared (IR) absorption spectroscopy. In short summary, substances with varying elements and molecular structures absorb frequencies of radiation that are resonant to it; and therefore the use of IR spectra can yield speciation of mixtures (i.e., relative amounts), as well as quantitative data such as the absolute concentrations.

Figure 1 is a schematic representation of our single droplet absorption spectroscopy experiment. Using the coordinate system from Figure 1, we can write the light extinction equations for a liquid and vapor in the following sections $[12,13]$. We also discuss the use of the Beer-Lambert equation for determining species concentration.

2.1. Liquid Scattering. The droplet investigated here has a high size parameter for a majority of its liquid existence and we predict complete attenuation due to light scattering:

$$
\chi \stackrel{\text { def }}{=} \frac{2 \pi r_{\text {droplet }}}{\lambda}
$$

where $\chi$ is the size parameter $[-] . r_{\text {droplet }}$ is the droplet radius, here $[\mathrm{nm}]$ but $[\mathrm{cm}]$ for later use. $\lambda$ is the wavelength of source light $[\mathrm{nm}]$.

That is to say, while the droplet is of diameter 1.90.19 [mm] over our optical frequency range of interest of $800-3400\left[\mathrm{~cm}^{-1}\right]$ (or 12,500-2,941 [nm]), the maximum and minimum size parameter are 2000-50, respectively. These sizes can be compared to the scattering regime graphic by Petty 2004 to show that scattering is at first a combination of geometric optics and Mie scattering [14]. As the droplet evaporates and the size parameter decreases, scattering is all Mie scattering for the droplet size we are considering. The scattering of light by large spherical shapes, like our droplet, is wavelength independent wherein the extinction efficiency can be calculated using size parameter and droplet refractive index.
Owing to the extinction paradox, extinction efficiency $\left(Q_{e}\right)$ has an oscillatory second-order response to size parameter where the steady state value is 2 [12]. Louedec and Urban show this behavior for a range of relative refractive indices (i.e., the index of the particle relative to the medium of 1.052.0) and size parameters up to 100 for nonabsorbing spherical particles.

We have absorbing spheres in our work where the real refractive indices can be found, but reliable sources for the imaginary indices (which represent absorption and dampen the oscillatory extinction efficiency behavior) have been elusive [14]. Nevertheless, ethanol and isooctane have real refractive indices of 1.31-1.35 and 1.33-1.44 over our range, respectively [15-17]. Considering these refractive indices, ethanol and isooctane also have $Q_{e}$ 's of 2 for a majority of our droplet lifetimes. $A Q_{e}$ of 2 means that, owing to the effects of diffraction, the droplet is opaque over twice the diameter of the geometric diameter. This background indicates that transmission of the IR light through the liquid to the detector will be zero and we neglect it. We verified this statement in the laboratory by probing an evaporating water droplet (index of refraction of 1.3 at 589 [nm]) using our FTIR method presented in this paper. We looked for liquid water IR absorption features. No liquid features were found, though vapor absorption was pervasive [18].

Since the droplet completely attenuates the incident light evenly across all wavelengths of interest, it is simply an offset in the absorption results where it was easily removed in postprocessing. Another consequence of the scattering is that the only light to reach the detector in our experiment will have travelled through the vapor. Therefore, we set our spatial integral limits for the vapor portion of the Beer-Lambert relationship from the droplet radius to the IR beam radius. It should be noted that while we could have set our integration limits to twice the geometric droplet radius because of our extinction efficiency, here we are using the geometric radius.

2.2. Vapor Absorption. The basic transmittance form of the Beer-Lambert relationship is given here:

$$
\left(\frac{I}{I_{0}}\right)_{\nu, y, \theta, t}=e^{-\sigma_{\nu} n_{y, \theta, t} L},
$$

where $I$ is the transmitted light $[\mathrm{W}] . I_{0}$ is the incident light [W]. $v$ is the optical frequency of interest $\left[\mathrm{cm}^{-1}\right] . y$ is the distance away from, and normal to, the centerline axis or axis of symmetry from Figure $1[\mathrm{~cm}] . \theta$ is the angle of revolution from Figure 1 [rad]. $t$ is time [s]. $\sigma_{v}$ is the absorption cross section $\left[\mathrm{cm}^{2} /\right.$ molecule]. $n$ is the number density [molecules $\left./ \mathrm{cm}^{3}\right] . L$ is path length.

It is apparent given the spatial and temporal dependencies of (2) that additional relationships and other details are required to capture the physical situation. We created the model here by reasoning what fraction of light of the FTIR detector would collect over our experiment as a function of time. The $D^{2}$ law was used to account for the time varying 
nature of the droplet size as well as the change in radial concentration of the vapor cloud over time [8]:

$$
D^{2}(t)=D_{0}^{2}-K t,
$$

where $D$ is diameter of droplet as a function of time [m]. $D_{0}$ is initial diameter $[\mathrm{m}] . K$ is evaporative constant $=$ $\left(8 \rho_{\text {air }} \mathscr{D}_{a b} / \rho_{\text {liq }}\right) \ln \left(1+B_{Y}\right)\left[\mathrm{m}^{2} / \mathrm{s}\right] . \rho$ is density $\left[\mathrm{kg} / \mathrm{m}^{3}\right] . \mathscr{D}_{a b}$ is diffusion coefficient $\left[\mathrm{m}^{2} / \mathrm{s}\right] . B_{Y}$ is transfer number $=\left(Y_{A, s}-\right.$ $\left.Y_{A, \infty}\right) /\left(1-Y_{A, s}\right) \cdot Y_{A, s}$ is vapor mass fraction at droplet surface. $Y_{A, \infty}$ is vapor mass fraction far from surface.

Assumptions for $D^{2}$ model include that the vapor cloud is not stagnant and diffuses radially (buoyancy of the gases is negligible) into the surrounding atmosphere and that evaporation occurs at a constant rate.

Initial conditions for (3) were determined experimentally as described later.

The surrounding vapor cloud profile varies according to

$$
Y(r, t)=1-\left(1-Y_{A, s}\right)^{r_{0}(t) / r},
$$

where $Y_{A, s}$, as with the $D^{2}$ law, is the mass fraction at the surface of droplet. This is determined with the mole fraction at the surface-by way of the Clausius-Clapeyron relationand knowledge of the molecular weights. This value is assumed constant over time. $r_{0}(t)$ is the initial radius of the droplet, which is assumed to evolve with time according to the $D^{2}$ law. $r$ is the radial location inside the vapor cloud. The outer radius of the vapor cloud was set to be where a $99 \%$ decay of the original mole fraction occurred. Since the liquid droplet is assumed to decay according to the $D^{2}$ evaporative model, every step in time results in a new $r_{0}$ value and therefore new outer radius value.

The $D^{2}$ law (3) governs the droplet size with time, and the vapor cloud distribution (4) alters with this droplet size as $Y_{A, s}$ is assumed constant. However, buoyancy is likely significant, and we expect some discrepancy between the model and results. In particular, we anticipate that the experimental absorbance results should be higher than that of the idealized model. Since the vapor cloud is significantly larger than the beam width for the lifetime of the droplet, buoyancy may cause the lower portion of the vapor cloud to rise into the beams path and thus increasing the overall number density. The light intensity distribution that probes the droplet and vapor cloud will now be introduced.

The mass fraction concentration profile was converted to mass density by way of the ideal gas law and combined with the original mass fraction profile to obtain a molar concentration profile.

Spatial information was first modeled in two dimensions assuming axisymmetry. More specifically, we used half of a Gaussian distribution for the incident light beam:

$$
I_{0}(y)=a e^{-(y-b)^{2} / 2 c^{2}},
$$

where $a$ is the amplitude of the beam. $c$ controls the width of the beam $[\mathrm{mm}] . b$ is the center location of the beam relative to a reference axis [mm].

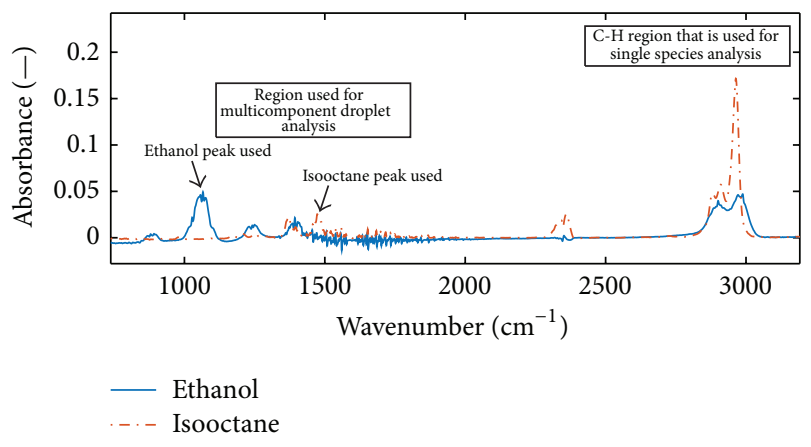

FIGURE 2: Spectra of isooctane and ethanol from this study where the absorbance peaks used to track composition are highlighted.

For the line of sight species of interest vapor concentration, which varies in $t$ and in coordinates $y$ and $r$ from Figure 1, we used an Abel transform [19]:

$$
n L(y, t)=\int_{0}^{R(t)} \frac{r n(r, t)}{\sqrt{y^{2}-r(t)^{2}}} d r,
$$

where $n L$ is the integrated number density along the line of sight [molecules $\left./ \mathrm{cm}^{2}\right] . R(t)$ is the radius of the vapor cloud to be considered in the Abel transform $[\mathrm{cm}]$.

Once the two-dimensional model was complete, we rotated it around the axis of symmetry to recreate the entire three-dimensional spatial portion of the model.

Given the preceding equations, we applied the BeerLambert relation to create our following model of the fraction of light collected by the detector at a given optical frequency and time:

$$
\left(\frac{I}{I_{0}}\right)_{\nu, y, \theta, t}=\frac{\int_{0}^{2 \pi} \int_{r_{\text {droplet }}}^{r_{\text {bea }}(t)} I_{0}(y) e^{-\sigma_{y} n L(y, t)} d y d \theta}{\int_{0}^{2 \pi} \int_{r_{\text {droplet }}(t)}^{r_{\text {beam }}} I_{0}(y) d y d \theta},
$$

where $r_{\text {beam }}$ is the FTIR light source radius [cm].

2.3. Absorption Spectra. Our absorbance spectra for ethanol and isooctane, shown in Figure 2, illustrate this principle in that the two substances have unique characteristic absorbing frequencies that the other lacks. In this example, ethanol has a unique absorbance band around $1100\left[\mathrm{~cm}^{-1}\right]$ and isooctane at $1500\left[\mathrm{~cm}^{-1}\right]$. As a result, with time-resolved spectra, it is possible to analyze these intrinsic absorption bands during droplet evaporation to investigate preferential evaporation.

\section{Materials and Methods}

The FTIR used for this work was a Thermo Scientific Nicolet iS10 shown schematically in Figure 3. The radiation source is a Mid-IR EverGlo ${ }^{\mathrm{Tw}}$ which is collimated unlike the high irradiance particle heating approach of others. The beam was split with a $\mathrm{KBr} / \mathrm{Ge}$ beam splitter which allows spectra to be collected in the $400-4000\left[\mathrm{~cm}^{-1}\right]$ range. This FTIR also has a deuterated triglycine sulfate detector. All experiments were conducted with an open beam path setup. The beam diameter 


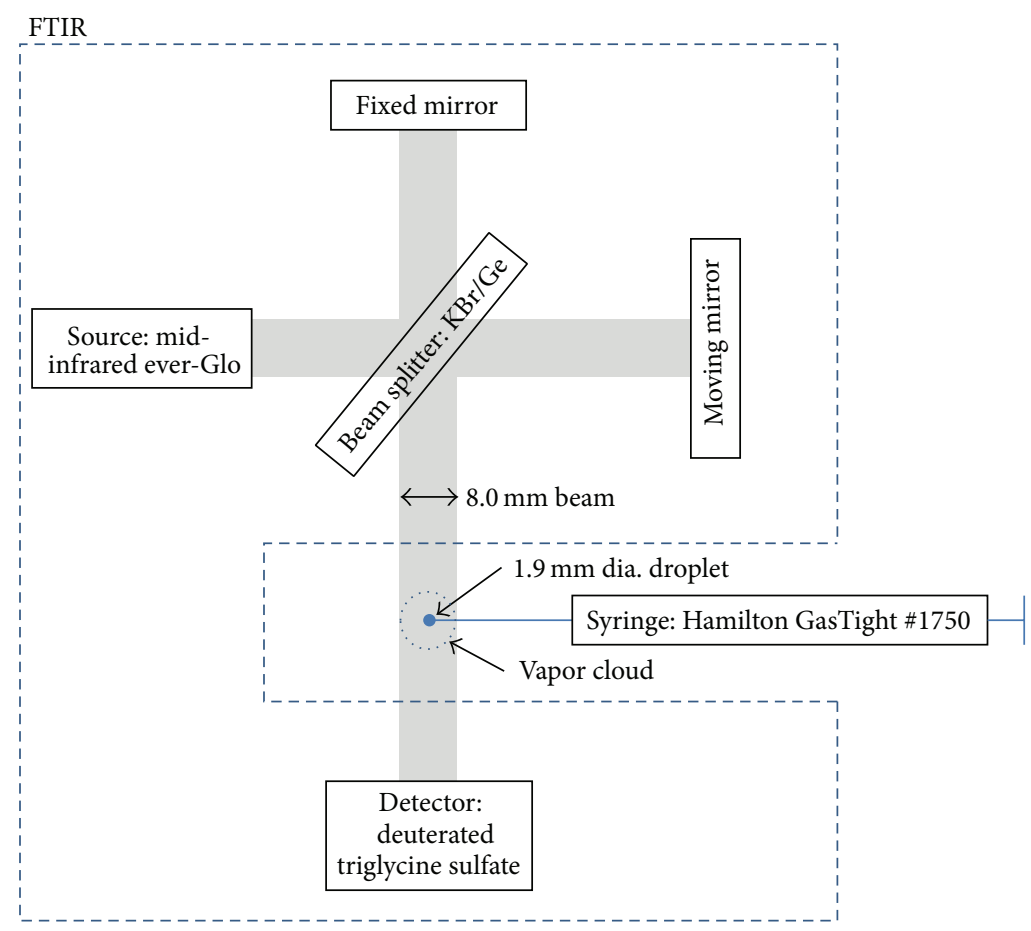

FIGURE 3: Experimental layout of droplet in FTIR beam path.

is not provided in the instrument documentation, so it was experimentally determined to be $8[\mathrm{~mm}$ ] by slowly moving a razor blade on a translation stage until the maximum magnitude of the interferogram was interrupted on both sides. The beam profile was also a curiosity; the beam was measured and had a Gaussian profile. The profile was determined through a similar experiment as used for determination of the beam width, wherein the beam was incrementally obstructed by a blade on a stage and the magnitude was recorded at each interval. The spectra samples were acquired at a resolution of $4\left[\mathrm{~cm}^{-1}\right]$ with two full spectrum scans per sample. All data were returned in a single beam format using $\mathrm{OMNIC}^{\mathrm{m}}$ software.

The suspension method for hanging a droplet in the beam path of an FTIR was accomplished using a Hamilton Gastight $500[\mu \mathrm{L}]$ Syringe with a $0.76[\mathrm{~mm}]$ diameter (22-gauge) blunt tipped needle. It was determined, through eight repeated observations, that an average droplet size of $4[\mu \mathrm{L}]$ could be suspended from the end of the needle. This was accomplished by plunging the liquid out of the syringe and releasing before the droplet mass was too large-that is, before the droplet separated from the needle. It was approximated that the suspending droplet was spherical in nature and therefore a $4[\mu \mathrm{L}]$ droplet would yield an approximate diameter of $1.9[\mathrm{~mm}]$.

For proper droplet orientation, the syringe was held by a stand such that the droplet center was coincident with the centerline of the FTIR reference helium neon ( $\mathrm{HeNe}$ ) laser (see Figure 3). It was assumed that this alignment would approximately center the droplet in the middle of the FTIR light source path. Single beam IR spectra were collected, wherein the background spectra $\left(I_{0}\right)$ had the needle slightly
TABLE 1: Selected wavenumber used to track species concentration in absorption spectra.

\begin{tabular}{|c|c|c|c|}
\hline & $\begin{array}{c}\text { Droplet } \\
\text { experiment }\end{array}$ & $\begin{array}{l}\text { Wavenumber } \\
\qquad\left[\mathrm{cm}^{-1}\right]\end{array}$ & $\begin{array}{c}\text { Absorption cross } \\
\text { section } \\
\times 10^{18} \\
{\left[\mathrm{~cm}^{2} / \text { molecule }\right]} \\
\text { vapor }\end{array}$ \\
\hline Isooctane & $\begin{array}{c}\text { Single } \\
\text { component }\end{array}$ & 2964 & 1.63 \\
\hline Ethanol & $\begin{array}{c}\text { Single } \\
\text { component }\end{array}$ & 2988 & 0.28 \\
\hline Isooctane & Mixture & 1479 & 0.23 \\
\hline Ethanol & Mixture & 1066 & 0.29 \\
\hline
\end{tabular}

protruding in the beam's path, followed by postprocessing via Matlab software. The code implemented the Beer-Lambert law so that time-resolved single beam spectra $(I)$ were converted to time-resolved absorbance (i.e., $\left.\ln \left[I / I_{0}\right]\right)$ spectra.

With the acquired absorbance spectra, a unique peak wavelength for each species was selected to be used to identify the relative gaseous concentrations of said species at a given time interval (see Table 1). The absorption cross sections used in our model are included from [20].

Single component measurements utilized the strongest absorbance for the best signal-to-noise ratio, which happened to be in overlapping spectral regions as seen in Table 1. Because of this overlap though, separate spectral regions were chosen for mixtures, as can be seen in Figure 2, such that preferential vaporization can be observed and quantified 
TABLE 2: Properties of liquid substances used for single droplet and binary droplet experiments.

\begin{tabular}{|c|c|c|c|c|c|c|}
\hline Species & Formula & $\begin{array}{l}\text { Density } \\
{\left[\mathrm{kg} / \mathrm{m}^{3}\right]}\end{array}$ & $\begin{array}{c}\text { Diffusion } \\
\text { coefficient } \\
\mathscr{D}_{a b} \\
\times 10^{5}\left[\mathrm{~m}^{2} / \mathrm{s}\right]\end{array}$ & $\begin{array}{l}\text { Boiling point } \\
{\left[{ }^{\circ} \mathrm{C}\right]}\end{array}$ & Vapor pressure $[\mathrm{kPa}]$ & Molar mass $[\mathrm{kg} / \mathrm{kmol}]$ \\
\hline Isooctane & $\mathrm{C}_{8} \mathrm{H}_{18}$ & 690 & 0.653 & 99 & 8.713 & 114.23 \\
\hline Ethanol & $\mathrm{C}_{2} \mathrm{H}_{6} \mathrm{O}$ & 789 & 1.108 & 78 & 5.27 & 46.07 \\
\hline
\end{tabular}

based on the magnitude of absorbance at a designated wavenumber.

Next, samples were taken with the needle (no droplet) being incrementally lowered into, and through, the beam. This test also showed no apparent wavelength dependent variability of the spectra; however, significant variability of water vapor and $\mathrm{CO}_{2}$ was noticed in the spectra, presumably from the operator's breath and variable laboratory conditions. A nitrogen purge was conducted to improve the signal-tonoise ratio of the absorbers of interest, that is, to reduce the absorption of nontarget species. This FTIR has a built-in port for nitrogen purging the unit itself; with the addition of a glove bag around the unit we were able to purge the entire setup and significantly reduce the amount of water vapor and $\mathrm{CO}_{2}$ being observed in the spectra samples.

Another problem that was addressed was the issue of capillary effects and seepage from the end of the needle. Initial data collection suggested that the droplet would never fully disperse, indicated by the characteristic absorbance peaks never completely reaching zero. To eliminate this concern, and in effort to include droplet temperature data, a thermocouple (type K with $0.81 \mathrm{~mm}$ [0.032 in] diameter, Omega TJ36-CASS-032U-6), of an equal diameter to the syringe, was used for suspending droplets. The droplets were carefully placed on the end of the thermocouple with the syringe. This approach proved to have significant benefits. First, there was no residual evaporation from the liquid in the needle seeping out. Second, we now had the ability to record the temperature profile of the droplets as they were evaporating. Note that evaporation diameter results, seen in Results, are entirely from the syringe whereas the temperature data results utilized the thermocouple. It should be noted that, unlike quartz fibers and electrodynamic levitation, both our needle and thermocouple suspension approaches introduce heat conduction concerns.

Ethanol and isooctane (2,2,4-trimethylpentane) were selected as components of a binary mixture because they are representative of components in oxygenated gasoline. The properties of the two components are listed in Table 2 [21].

The diffusion coefficient was found in dry air [8]. A mixture of $85 \%$ isooctane and $15 \%$ ethanol by volume was chosen as the mixture properties to be representative of E15 oxygenated gasoline in the United States. The results were averaged over five separate droplet evaporation instances.

\section{Results and Discussion}

4.1. Single Component Droplet Evaporation by IR Absorption Spectroscopy. The single species droplet absorption spectra

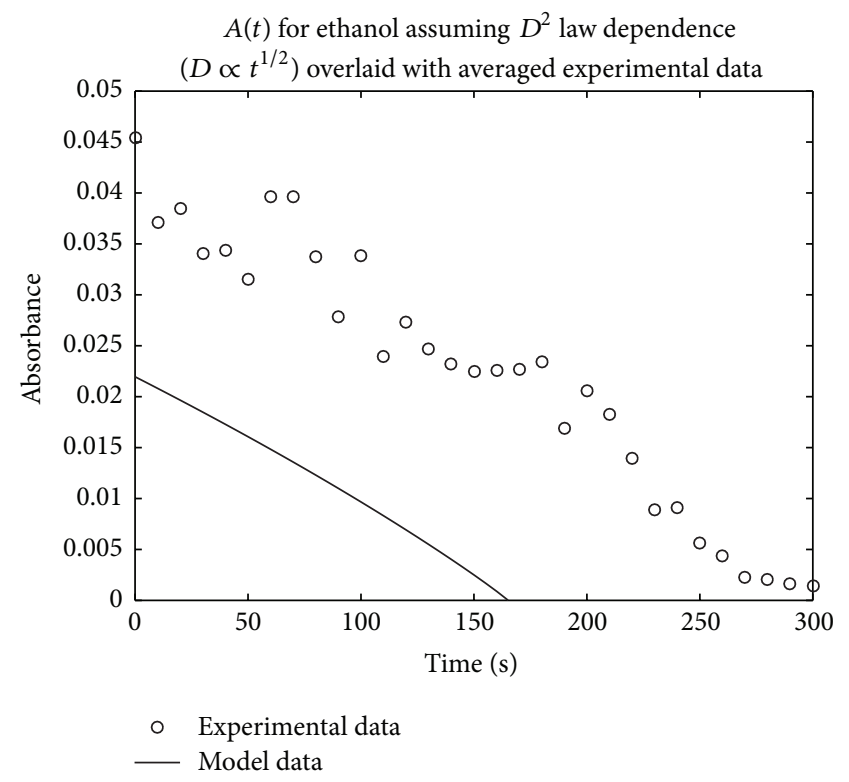

FIGURE 4: Single component droplet evaporation comparing the experimental data from IR absorption spectroscopy of our model for $100 \%$ ethanol. Data shown were averaged over five experiments.

over time were compared to our model (i.e., (7)). The absorption is seen to decrease over time; however there was some variability in that the spectra did not decrease smoothly and monotonically with each time interval due to nonideal effects. It can be seen in Figures 4 and 5 that the averaged data resemble our model, despite the nonideal influences on the final results.

The decay times are similar in both the model and the empirical data. It seems that our measurement results are indicative of the reduction over time of the actual droplet diameter, but perhaps nonideal effects prevent the initial conditions from matching. The disagreement in magnitudes at time zero may also be attributed to mischaracterization of the initial droplet diameter. This is in spite of our efforts to characterize the initial diameter through multiple trials as previously mentioned.

\subsection{Single and Binary Droplet Evaporation by Thermocouple.} In our work, the temperature profiles of isooctane and ethanol exhibit nonmonatomic behavior as also seen in smaller diameter droplet $(0.015 \mathrm{~mm}$ diameter $)$ modeling work done by Camm et al. [10]. Camm et al. found that a wellmixed $60 \%$ isooctane and $40 \%$ ethanol by volume (i.e., E40) 


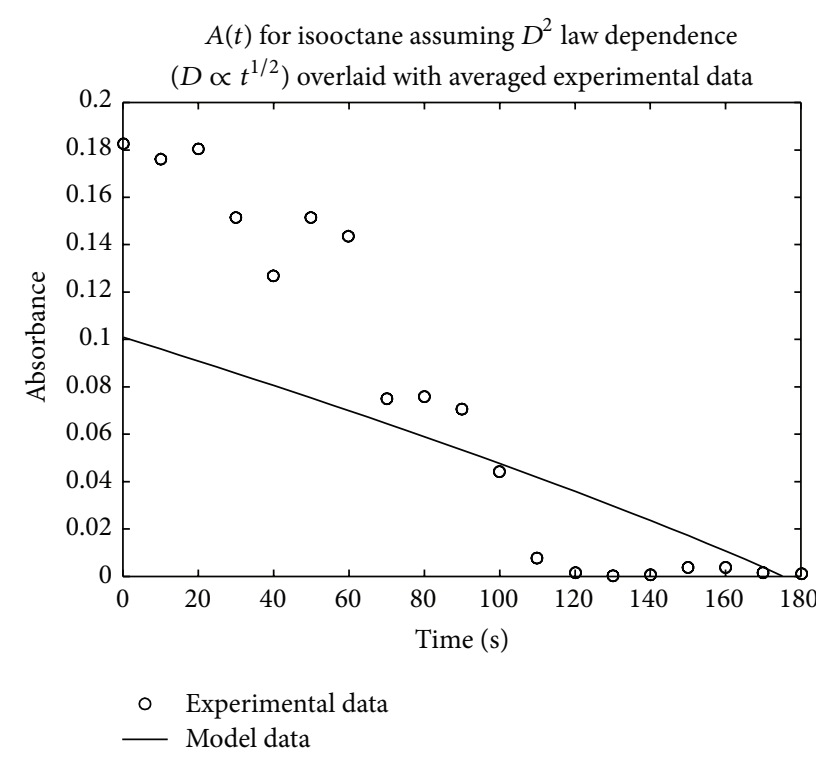

FIGURE 5: Single component droplet evaporation comparing the experimental data from IR absorption spectroscopy of our model for $100 \%$ isooctane. Data shown were averaged over five experiments.

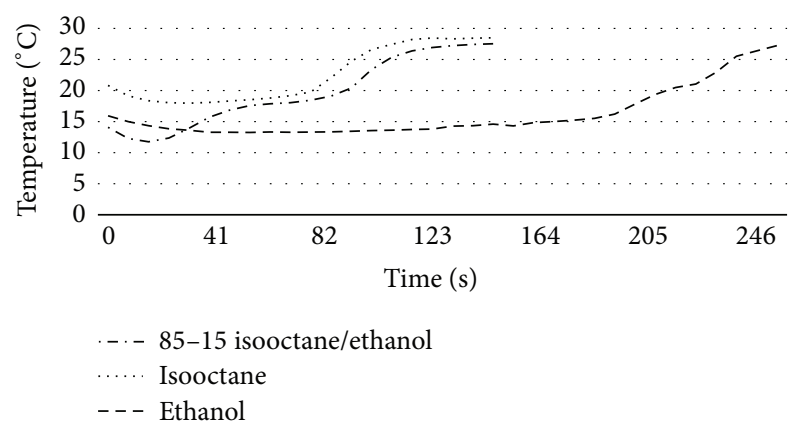

FIGURE 6: Droplet evaporation by suspension from a thermocouple and the corresponding thermocouple measurements over time.

mixture droplet completed its temperature-time profile in a manner similar to isooctane.

In Figure 6, the ethanol temperature profile takes longer to equilibrate relative to isooctane and mixture of isooctane and ethanol. Considering the single component absorbance spectra, it can be seen that the absorbance for ethanol also takes longer in relation to isooctane.

Considering the binary mixture temperature-time history in Figure 6, primarily consisting of isooctane, it was expected that the temperature profile would exhibit nearly the same profile as pure isooctane. However, it can be seen that there is a more significant cooling effect early in the evaporation process (i.e., a lower temperature near time zero), more so than pure ethanol alone. This phenomenon may suggest preferential evaporation along with a synergistic blending (i.e., the mixture has a greater cooling effect than either of the individual components alone) effect for faster evaporation. However, as with the work of Camm et al., at longer times the temperature profile of the mixture behaves similarly to that of neat isooctane [10].

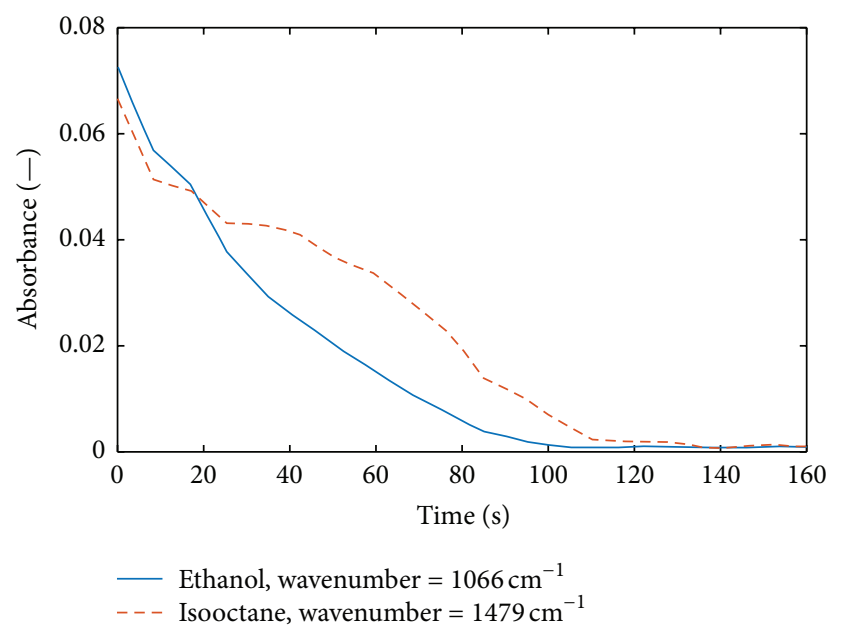

Figure 7: Binary droplet ( $85 \%$ isooctane and $15 \%$ ethanol by volume) evaporation measured by IR absorbance.

Note that a third method for evaporation-time measurement, a time series of digital photographs of the droplet diameter with a grid background, was also conducted, but the images were not of sufficient quality for reliable interpretation of droplet diameter. As a reminder, the approaches to verifying the use of diameter over time were a means to verify the next approach which is tracking individual species evaporation over time for a multicomponent fuel using spectral properties.

4.3. Binary Mixture Droplet Evaporation by IR Absorption Spectroscopy. The experimental binary absorbance spectra results are shown in Figure 7 which are not compared to our single component model. Focusing on isooctane in the mixture, it is seen that the isooctane diminishes at a faster rate initially than neat isooctane from Figure 5. In Figure 5, the absorbance of neat isooctane decreased slowly at first then more rapidly (i.e., decreasing concave down).

However, the binary mixture yields a short rapid decrease initially, then slows, decreases more rapidly again, and finally approaches zero (i.e., exponential decay). It appears that the isooctane could have a two-stage diminishing profile or, as described by Hallett and Beauchamp-Kiss, the presence of the polar ethanol molecule negates Raoult's law behavior thereby prolonging the evaporation of the isooctane [5]. However, it should be noted that this approach is in development and though broad trends can be interpreted, finer structure could be variation due to a poor signal-to-noise ratio rather than physical behavior.

Considering ethanol, it can be seen that the absorbance also decays rapidly and resembles an exponential decaying profile, in comparison to neat ethanol's slower initial decay. This would explain the lower temperature in the blended mixture initially. The overall time for ethanol to decay in the binary mixture decreased over twofold in comparison to its neat component results. This, along with the fact that the overall time for isooctane to diminish was relatively unchanged, lends some evidence to ethanol as a preferential 
evaporation pathway. That is to say, ethanol appears to evaporate more quickly in the presence of isooctane in the mixture ratios shown here than as a pure component.

\section{Conclusion}

Infrared absorption, by way of FTIR, and a thermocouple were used to track the evaporation of single component and binary mixtures droplets. The single component absorbance data correlated with our model and provided a means to explain the observed temperature profiles. The absorbance data in the binary mixture shows that there is significant increase in the evaporation of ethanol compared to ethanol alone. Neat ethanol demonstrated a slow, linear diminishment, but when blended with isooctane it diminished significantly faster overall and at an exponential rate. For isooctane, a faster absorbance decay rate occurred initially, but the total time for evaporation is nearly unchanged. Because of these trends, it is believed that the ethanol is more preferential for evaporation and there are synergistic blending effects present.

With the uncertainty of the equipment due to low signalto-noise ratio, as well as nonideal effects in the data, development is ongoing. However, it is definite that the IR spectroscopic analysis technique presented here deserves continued attention. By including nonideal effects into the model and increasing the signal-to-noise ratio of the absorbance spectra (e.g., by averaging more experimental runs) it appears plausible to observe preferential evaporation of binary, and even more than binary, multicomponent substances. With more detailed modeling and slight experimental technique alterations, this novel technique may be the means to quantitatively improve our understanding of preferential evaporation of multicomponent mixtures.

\section{Conflict of Interests}

The authors declare that there is no conflict of interests regarding the publication of this paper.

\section{Acknowledgments}

The authors thank Dr. Jeff Gautschi for generous use of his lab space and use of the FTIR. Oregon State University, Cascades, supports this work.

\section{References}

[1] P. Hottenbach, T. Brands, and G. Grünefeld, "An experimental investigation on the evaporation characteristics of a twocomponent fuel in diesel-like sprays," SAE International Journal of Engines, vol. 4, no. 1, pp. 800-812, 2011.

[2] Y. Ra and R. D. Reitz, "A vaporization model for discrete multicomponent fuel sprays," International Journal of Multiphase Flow, vol. 35, no. 2, pp. 101-117, 2009.

[3] J. B. Wei, B. D. Shaw, and J. S. Xiao, "Transient measurements of the composition of evaporating droplets by fiber optic absorption spectroscopy," Optics and Lasers in Engineering, vol. 39, no. 1, pp. 91-108, 2003.

[4] H. Ghassemi, S. W. Baek, and Q. S. Khan, "Experimental study on binary droplet evaporation at elevated pressures and temperatures," Combustion Science and Technology, vol. 178, no. 6, pp. 1031-1053, 2006.

[5] W. L. H. Hallett and S. Beauchamp-Kiss, "Evaporation of single droplets of ethanol-fuel oil mixtures," Fuel, vol. 89, no. 9, pp. 2496-2504, 2010.

[6] G. S. Grader, S. Arnold, R. C. Flagan, and J. H. Seinfeld, "Fourier transform infrared spectroscopy of a single aerosol particle," The Journal of Chemical Physics, vol. 86, no. 11, pp. 5897-5903, 1987.

[7] S. Arnold, E. K. Murphy, and G. Sageev, "Aerosol particle molecular spectroscopy," Applied Optics, vol. 24, no. 7, pp. 1048-1053, 1985.

[8] S. R. Turns, An Introduction to Combustion: Concepts and Applications, McGraw-Hill, New York, NY, USA, 2012.

[9] S. Raghuram and V. Raghavan, "Thermodynamic analysis of evaporation of levitated binary and ternary liquid fuel droplets under normal gravity," ISRN Thermodynamics, vol. 2012, Article ID 167281, 10 pages, 2012.

[10] J. Camm, R. Stone, M. H. Davy, and D. Richardson, “The modelling of multi-component droplet evaporation," in Proceedings of the 23rd "Journées d'Etude" of the Belgian Section of the Combustion Institute, Brussels, Belgium, May 2014.

[11] R. Hołyst, M. Litniewski, D. Jakubczyk et al., "Evaporation of freely suspended single droplets: experimental, theoretical and computational simulations," Reports on Progress in Physics, vol. 76, no. 3, Article ID 034601, 2013.

[12] C. F. Bohren and D. R. Huffman, Absorption and Scattering of Light by Small Particle, John Wiley \& Sons, New York, NY, USA, 2008.

[13] K. N. Rao, Molecular Spectroscopy: Modern Research, Academic Press, London, UK, 1976.

[14] G. W. Petty, A First Course in Atmospheric Radiation, Sundog Publishing, LLC, Madison, Wis, USA, 2004.

[15] fractiveindex.info, fractiveindex.info, December 2015, http:// refractiveindex.info/.

[16] S. Kedenburg, M. Vieweg, T. Gissibl, and H. Giessen, "Linear refractive index and absorption measurements of nonlinear optical liquids in the visible and near-infrared spectral region," Optical Materials Express, vol. 2, no. 11, pp. 1588-1611, 2012.

[17] M. R. Anderson, Determination of infrared optical constants for single component hydrocarbon fuels [M.S. thesis], University of Missouri-Rolla, 2000.

[18] Thermo Fisher Scientific, OMNIC Specta General Chemicals Software Version 9.2.98, Thermo Fisher Scientific, Waltham, Mass, USA, 2012.

[19] A. W. Caswell, Water vapor absorption thermometry for practical combustion applications [Ph.D. thesis], University of WisconsinMadison, Madison, Wis, USA, 2009.

[20] S. W. Sharpe, T. J. Johnson, R. L. Sams, P. M. Chu, G. C. Rhoderick, and P. A. Johnson, "Gas-phase databases for quantitative infrared spectroscopy," Applied Spectroscopy, vol. 58, no. 12, pp. 1452-1461, 2004.

[21] R. Stenutz, “Tables for Chemistry,” December 2009, http://www .stenutz.eu/chem. 

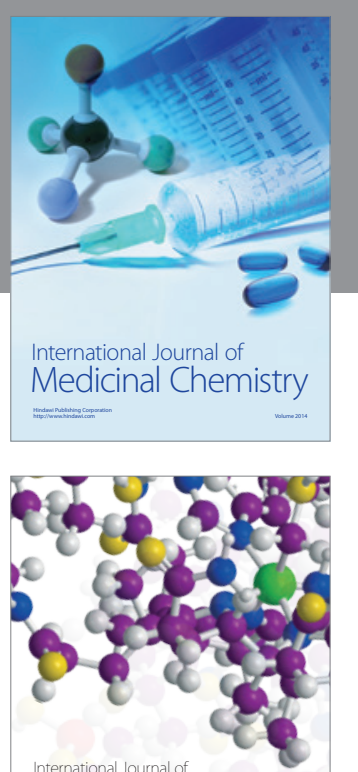

Carbohydrate Chemistry

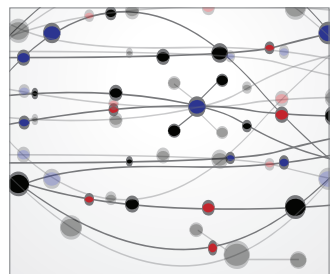

The Scientific World Journal
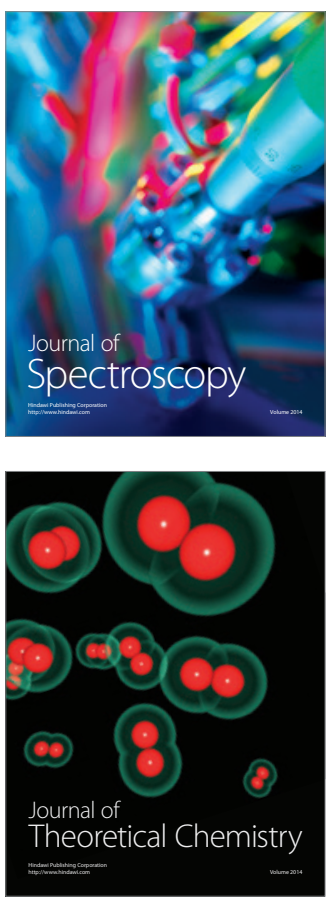
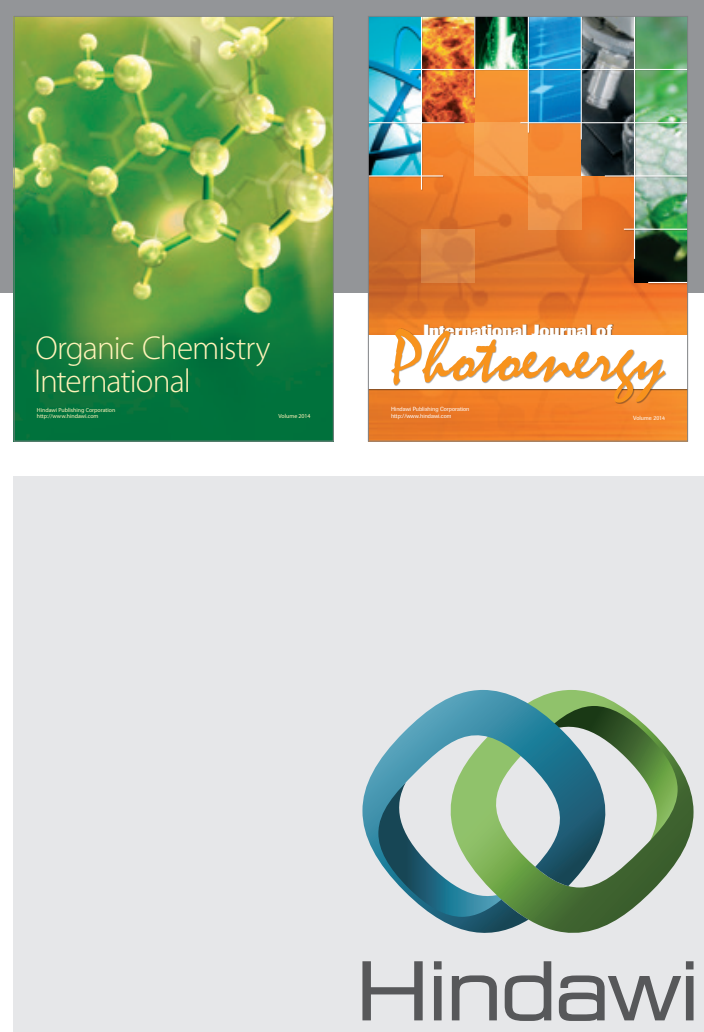

Submit your manuscripts at

http://www.hindawi.com

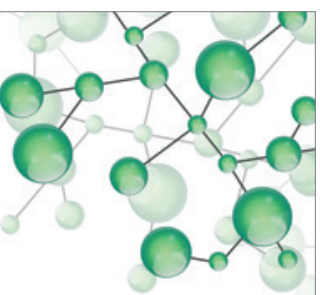

International Journal of

Inorganic Chemistry

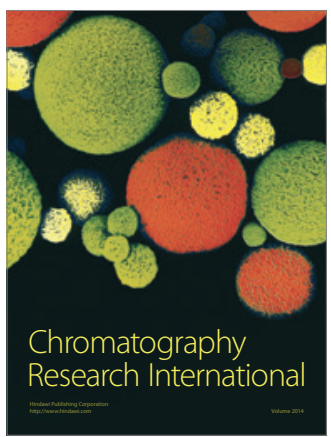

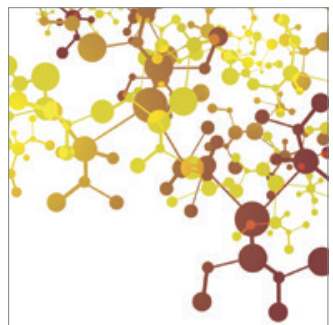

Applied Chemistry
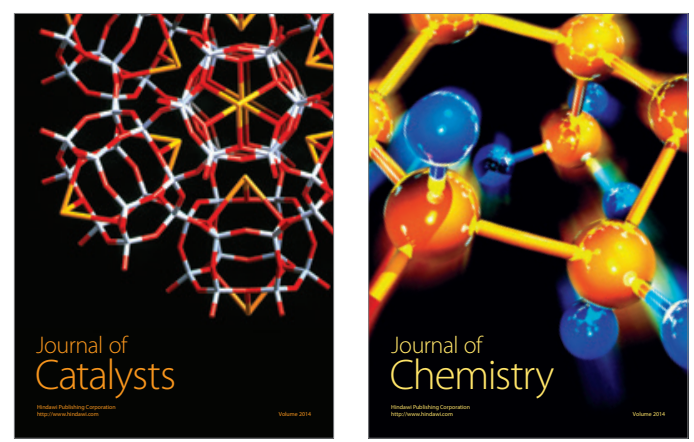
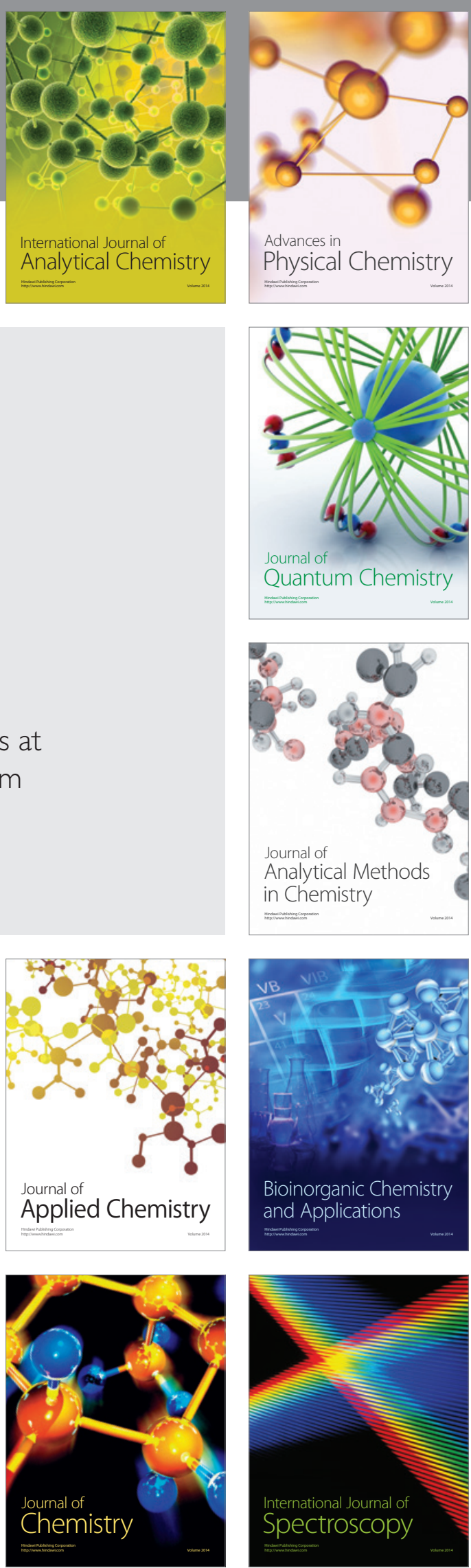